\title{
Germline DNA copy number variation in individuals with Argyrophilic grain disease reveals CTNS as a plausible candidate gene
}

\author{
Darine Villela ${ }^{1}$, Lilian Kimura ${ }^{1}$, David Schlesinger ${ }^{2}$, Amanda Gonçalves ${ }^{3}$, Peter L. Pearson ${ }^{1}$, \\ Claudia K. Suemoto ${ }^{4}$, Carlos Pasqualucci ${ }^{5}$, Ana Cristina Krepischi ${ }^{3}$, Lea T. Grinberg ${ }^{5}$ and Carla Rosenberg ${ }^{1}$ \\ ${ }^{1}$ Departamento de Genética e Biologia Evolutiva, Instituto de Biociências, Universidade de São Paulo, \\ São Paulo, SP, Brazil. \\ ${ }^{2}$ Instituto Israelita de Ensino e Pesquisa Albert Einstein, São Paulo, SP, Brazil. \\ ${ }^{3}$ Instituto Nacional de Ciência e Tecnologia em Oncogenômica, Hospital AC Camargo, São Paulo, \\ SP, Brazil. \\ ${ }^{4}$ Departamento de Medicina Interna, Faculdade de Medicina da Universidade de São Paulo, \\ São Paulo, SP, Brazil. \\ ${ }_{5}^{5}$ Departamento de Patologia, Banco de Encéfalos Humanos do Grupo de Estudos em Envelhecimento \\ Cerebral, Faculdade de Medicina, Universidade de São Paulo, São Paulo, SP, Brazil.
}

\begin{abstract}
Argyrophilic grain disease (AGD) is a progressive neurodegenerative disease of the human brain that has never been associated to a particular gene locus. In the present study, we report the results of a CNV investigation in 29 individuals whose anatomopathologic investigation of the brain showed AGD. Rare CNVs were identified in six patients $(21 \%)$, in particular a $40 \mathrm{~kb}$ deletion at 17 p13.2 encompassing the CTNS gene. Homozygote mutations in CTNS are known to cause cystinosis, a disorder characterized by the intralysosomal accumulation of cystine in all tissues. We present the first CNV results in individuals presenting AGD and a possible candidate gene implicated in the disorder.
\end{abstract}

Keywords: Argyrophilic grain disease, copy number variations, CNVs, array-CGH, CTNS.

Received: May 16, 2013; Accepted: October 8, 2013.

Argyrophilic grain disease (AGD) is a neurodegenerative disorder of the aged human brain morphologically characterized by the presence of argyrophilic grains (AG) mainly in limbic areas. Several studies have shown frequent association of AGD with other tauopathies such as Alzheimer's and Pick's diseases, and its prevalence increases significantly with age, present in up to $40 \%$ of all 85 years or older individuals (Tolnay and Clavaguera, 2004). However, the cause of AGD remains unknown; the disease seems to be sporadic but genetic studies have failed to discover a link between AGD and a particular gene locus.

The contribution of DNA copy number variations (CNVs) in the phenotypes of various complex diseases has been widely demonstrated over the past years. In fact, CNVs are increasingly recognized to be a prevalent form of common genetic variation in the human population. Even though a great number of studies have demonstrated the role of $\mathrm{CNVs}$ in the etiology of several neuropsychiatric

Send correspondence to Carla Rosenberg. Department of Genetics and Evolutionary Biology, Institute of Biosciences, University of São Paulo, Rua do Matão 277, 05508-090, São Paulo-SP, Brazil. E-mail: carlarosenberg@uol.com.br. disorders (Lee and Lupski, 2006; Cook and Scherer, 2008) there are no reports of specific CNVs related do AGD. Here, we identified rare constitutive CNVs in individuals with AGD, in particular one at 17p13.2 that points to deletions in the cystinosin, lysosomal cystine transporter gene, CTNS, as strong candidate to cause AGD.

The Brain Bank of the Brazilian Aging Brain Study Group (BBBABSG) (Grinberg et al., 2007) provided DNA from blood samples of 29 individuals with AGD. The individuals of our group ranged in age from 50 to 89 and included females and males. Table 1 presents the characterization of all cases investigated in this study. The neuropathological diagnosis of AGD was based, at least, on the presence of AG in hippocampal CA1 area (CA - Cornu Ammonis) and entorhinal region, as well as in pretangles in hippocampal CA2 area in sections immunostained with the phosphor-tau antibody (PHF1, 1:1000, gift from Peter Davies, NY) according to accepted criteria (Braak and Braak, 1987). The subject's clinical and functional statuses were assessed through a knowledgeable informant based on a validated clinical protocol. The protocol includes a series of semi-structured scales and questionnaires that cover ma- 
Table 1 - Case profiles of the all subjects with Argyrophilic grain disease investigated in this study.

\begin{tabular}{|c|c|c|c|c|c|c|c|}
\hline \multirow[t]{2}{*}{ Case } & \multirow{2}{*}{$\begin{array}{l}\text { Age at death } \\
\text { (years) }\end{array}$} & \multirow[t]{2}{*}{ Gender } & \multicolumn{3}{|c|}{ Stages of senile changes } & \multirow[t]{2}{*}{ Additional pathology } & \multirow[t]{2}{*}{ Cause of death } \\
\hline & & & NFT & $\mathrm{SP}$ & LB & & \\
\hline 1 & 85 & $\mathrm{~F}$ & 3 & A & - & - & Chronic obstructive pulmonary disease \\
\hline 2 & 89 & $\mathrm{~F}$ & 2 & 0 & - & VD & Pulmonary thromboembolism \\
\hline 3 & 85 & $\mathrm{~F}$ & 3 & $\mathrm{~B}$ & - & - & Esophageal cancer \\
\hline 4 & 84 & $\mathrm{~F}$ & 0 & 0 & - & - & Acute pulmonary edema \\
\hline 5 & 78 & M & 2 & A & - & VD & Peritonitis \\
\hline 6 & 85 & $\mathrm{~F}$ & 2 & 0 & - & - & Aortic aneurysm \\
\hline 7 & 79 & M & 0 & 0 & - & - & Gastric ulcer \\
\hline 8 & 82 & $\mathrm{~F}$ & 2 & 0 & - & - & Aortic aneurysm \\
\hline 9 & 72 & $\mathrm{~F}$ & 1 & 0 & - & VD & Ischemic heart disease \\
\hline 10 & 75 & $\mathrm{~F}$ & 2 & \multicolumn{2}{|c|}{$\mathrm{B}$} & $\mathrm{PD}$ & Pulmonary thromboembolism \\
\hline 11 & 67 & $\mathrm{~F}$ & 1 & 0 & - & - & Pneumonia \\
\hline 12 & 76 & $\mathrm{~F}$ & 3 & 0 & - & - & Heart failure \\
\hline 13 & 80 & M & 3 & A & - & - & Pneumonia \\
\hline 14 & 73 & $\mathrm{~F}$ & 3 & 0 & - & - & Myocardial sclerosis \\
\hline 15 & 81 & $\mathrm{~F}$ & 4 & $\mathrm{C}$ & - & $\mathrm{AD}+\mathrm{VD}$ & Aortic aneurysm \\
\hline 16 & 59 & M & 1 & 0 & - & - & Pneumonia \\
\hline 17 & 80 & M & 2 & 0 & - & - & Hypertensive heart disease \\
\hline 18 & 86 & M & 4 & 0 & - & - & Heart failure \\
\hline 19 & 58 & M & 1 & 0 & - & - & Coronary artery disease \\
\hline 20 & 50 & M & 1 & 0 & - & - & Pneumonia \\
\hline 21 & 76 & $\mathrm{~F}$ & 0 & 0 & - & - & Perforated gastric ulcer \\
\hline 22 & 76 & M & 0 & 0 & - & - & Myocardial infarction \\
\hline 23 & 80 & M & 0 & 0 & - & - & Myocardial infarction \\
\hline 24 & 88 & $\mathrm{~F}$ & 3 & 0 & - & - & Ischemic heart disease \\
\hline 25 & 69 & $\mathrm{~F}$ & 1 & 0 & - & - & Pulmonary thromboembolism \\
\hline 26 & 83 & $\mathrm{~F}$ & 3 & A & - & - & Myocardial infarction \\
\hline 27 & 85 & $\mathrm{~F}$ & 3 & 0 & - & - & Pneumonia \\
\hline 28 & 89 & M & 2 & 0 & - & - & Duodenal ulcer bleeding \\
\hline 29 & 80 & M & 3 & A & - & - & Chronic obstructive pulmonary disease \\
\hline
\end{tabular}

NFT=neurofibrillary tangle, Braak stage; $\mathrm{SP}=$ senile plaque, CERAD (Consortium to Establish a Registry for Alzheimer's Disease); LB=Lewy body; $\mathrm{VD}=$ vascular dementia; $\mathrm{PD}=$ Parkinson disease; $\mathrm{AD}=$ Alzheimer's disease.

jor functional abilities and were validated for assessment with an informant (Grinberg et al., 2007). BBBABSG's procedures were approved by the Ethical Board of University of São Paulo Medical School and the next-of-kin agreed to participate and signed an informed written consent.

To identify CNVs we performed oligonucleotide comparative genomic hybridization based on microarrays (array-CGH) using a whole-genome platform containing $\sim 180.000$ oligonucleotides (180k platform) (Oxford Gene Technologies, UK). Briefly, samples were labeled with Cy3- and Cy5-deoxycytidine triphosphates by random priming. Purification, hybridization and washing were carried out as previously reported (Krepischi et al., 2010). Scanned images of the arrays were processed using Feature Extraction software and data were analyzed with the
Genomic Workbench software, both from Agilent Technologies. To distinguish CNVs, we used the Aberration Detection Method 2 statistical algorithm (ADM2) with a sensitivity threshold of 6.7. A genomic segment was considered duplicated or deleted when the $\log _{2}$ ratio of the Test/Reference fluorescent intensities of a given region encompassing at least three probes was above 0.3 or below -0.3 , respectively. Detected CNVs were compared to CNV data from oligoarray studies documented in the Database of Genomic Variants (DGV). The relevant CNVs were validated by dye-swap hybridizations, and only a mirror result would be confirmatory of the CNVs presence.

The array-CGH analysis revealed rare $\mathrm{CNVs}$ (rare defined as frequency $<0.1 \%$ of population, based on DGV) in six individuals among the 29 with AGD, none of them re- 
current. To exclude that these rare CNVs represent common variants in the Brazilian population, we compiled CNV data obtained with the same $180 \mathrm{~K}$ array-CGH platform from more than 400 independent samples studied in our laboratory for reasons other than dementia. None of the rare CNVs documented in this study were detected in these subjects. Table 2 summarizes the rare $\mathrm{CNV}$ s identified in our group and shows the genes encompassed by these genomic imbalances. Any of the rare CNVs detected are potential candidates for the investigated phenotype; however, we highlighted the genomic $40 \mathrm{~kb}$ microdeletion of at 17 p13.2 that includes the CTNS gene as especially interesting due to its relevant gene content and the lack of reports on copy number changes affecting this gene. Figure 1 shows the validation of this CNV made by reverse labeling hybridization, where it is possible to see that the alteration is mirrored.

Table 2 - Descriptions of all rare copy number variations identified in the individuals with Argyrophilic grain disease.

\begin{tabular}{lccccccl}
\hline Case & Chr & Cytoband & Start site & End site & CNV type & Size $(\mathrm{kb})$ & Gene (s) \\
\hline 2 & 10 & $\mathrm{p} 13$ & 14996608 & 15043743 & del & 44 & MEIG1 \\
3 & 9 & $\mathrm{p} 24.1$ & 5763235 & 5881920 & del & 70 & KIAA1432, ERMP1 \\
$\mathbf{4}$ & $\mathbf{1 7}$ & $\mathbf{p} 13.2$ & $\mathbf{3 5 2 7 6 2 8}$ & $\mathbf{3 5 6 0 1 1 8}$ & del & $\mathbf{4 0}$ & SHPK, CTNS \\
14 & 1 & $\mathrm{p} 31.1$ & 78594557 & 79456374 & dup & 789 & GIPC2, MGC27382, PTGFR, IFI44L, ELTD1 \\
17 & 12 & $\mathrm{q} 12.1$ & 25723651 & 25755485 & del & 30 & IFLTD1 \\
21 & 5 & $\mathrm{q} 14.2-\mathrm{q} 14.3$ & 82630761 & 82851414 & dup & 103 & XRCC4, VCAN \\
\hline
\end{tabular}

Genomic positions based on GRCh37 Build reference sequence. Highlighted in bold is a CNV with relevant gene content for the investigated phenotype. $\mathrm{CNV}=$ copy number variation; $\mathrm{del}=$ deletion; dup $=$ duplication .

(A)

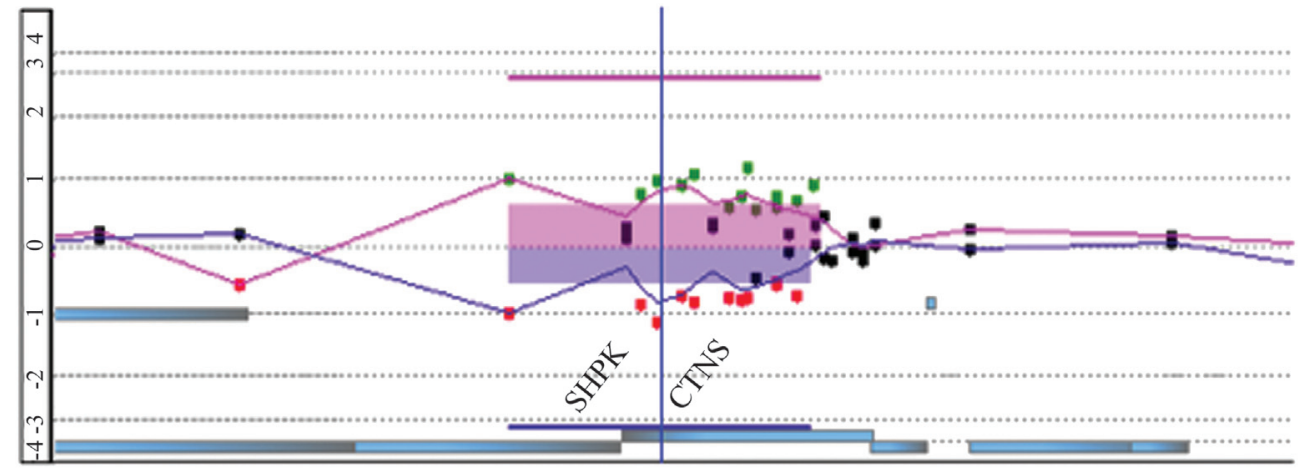

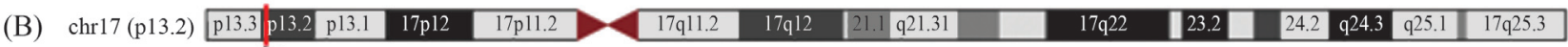

(C)

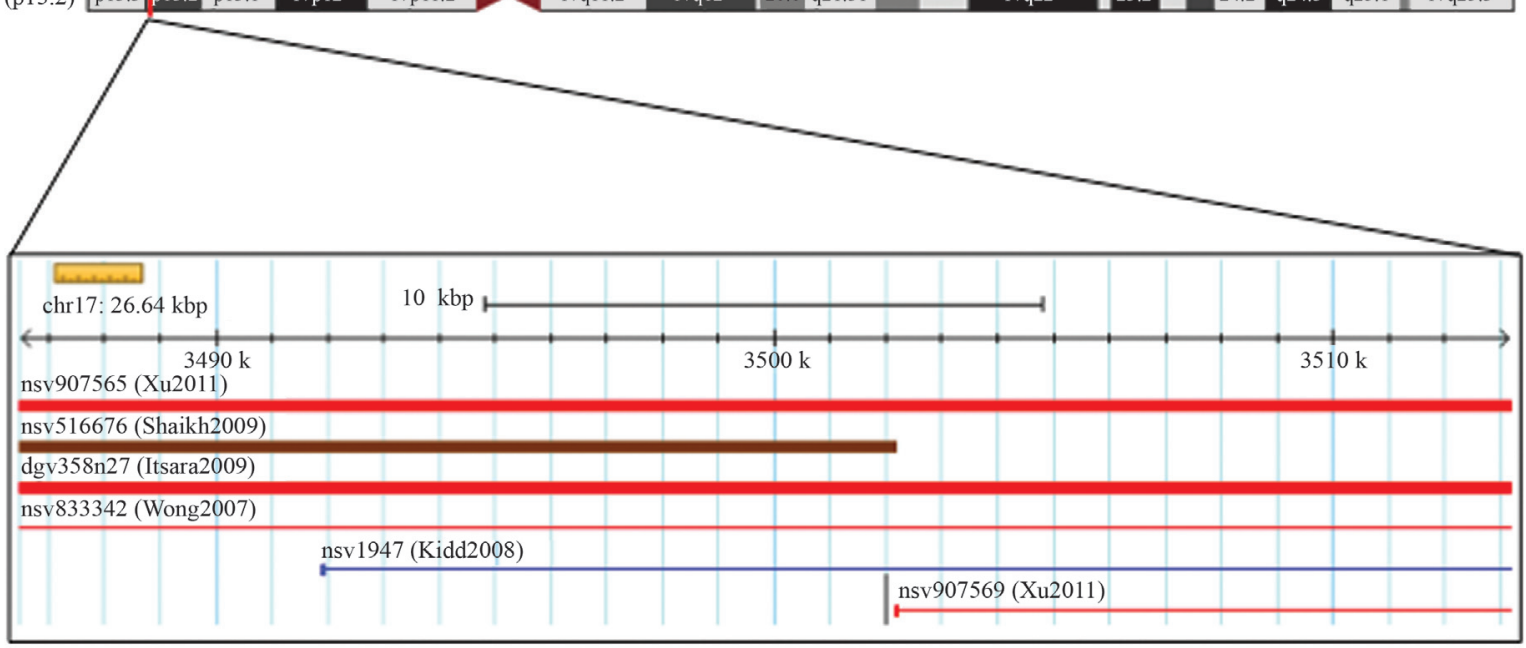

Figure 1 - The 17p13.2 rare CNV detected in a subject with Argyrophilic grain disease. (A) Array-CGH profile of the genomic segment containing the $\mathrm{CNV}$, and its reverse labeling hybridization (image based on the Genome Workbench software); (B) Ideogram of chromosome 17 showing the position of the CNV (small vertical red bar); (C) Region covered by the CNV. CNV loci reported in the general population are indicated by blue (gain), red (loss) and brown (complex rearrangement) bars in the Database of Genomic Variants (DGV) (image derived from the DGV, freeze September 2013). 
The CTNS gene encodes a seven-transmembrane domain protein that functions to transport cystine out of lysosomes. Its activity is driven by the $\mathrm{H}^{+}$electrochemical gradient across the lysosomal membrane. Mutations in this gene cause cystinosis, a rare autosomal recessive disorder characterized by the intralysosomal accumulation of cystine in all tissues (Kalatzis and Antignac, 2002). The most common mutation associated with this rare disease is a deletion of $65 \mathrm{~kb}$ presented in homozygosity that also includes the SHPK gene. The protein encoded by this latter gene has weak homology to several carbohydrate kinases, a class of proteins involved in the phosphorylation of sugars as they enter the cell, inhibiting return across the cell membrane (Wamelink et al., 2008). Our results show a heterozygous deletion in the CTNS gene encompassing the region of this common mutation associated with cystinosis. Literature data demonstrate that the brain is one of the last organs to be affected by the progressive cystine accumulation (Cochat et al., 1986). Cognitive impairments have been documented in some cystinosis patients, which presented deficit in visual-spatial memory (Trauner et al., 1988; Scarvie et al., 1996). Additionally, an investigation showed that Ctns -/- knockout mice present a severe age-related memory deficit (Maurice et al., 2009). Therefore, this evidence makes CTNS a good candidate gene for susceptibility to AGD.

In conclusion, this is the first study to identify a rare $\mathrm{CNV}$ at 17p13.2 with AGD and links this disease with a particular gene locus, the CTNS.

\section{Acknowledgments}

This work was supported by a FAPESP grant CR (2009/00898-1), and DV was supported by a FAPESP PhD fellowship (2010/15503-0). We thank the Brazilian Aging Brain Study Group for providing DNA samples.

\section{References}

Braak H and Braak E (1987) Argyrophilic grains: Characteristic pathology of cerebral cortex in cases of adult onset dementia without Alzheimer changes. Neurosci Lett 76:124-127.
Cochat P, Drachman R, Gagnadoux MF, Pariente D and Broyer M (1986) Cerebral atrophy and nephropathic cystinosis. Arch Dis Childhood 61:401-403.

Cook Jr EH and Scherer SW (2008) Copy-number variations associated with neuropsychiatric conditions. Nature 455:919923.

Grinberg LT, Ferretti RE, Farfel JM, Leite R, Pasqualucci CA, Rosemberg S, Nitrini R, Saldiva PH and Filho WJ (2007) Brain bank of the Brazilian aging brain study group - A milestone reached and more than 1,600 collected brains. Cell Tissue Banking 8:151-162.

Kalatzis V and Antignac C (2002) Cystinosis: From gene to disease. Nephrol Dial Transplant 17:1883-1886.

Krepischi AC, Knijnenburg J, Bertola DR, Kim CA, Pearson PL, Bijlsma E, Szuhai K, Kok F, Vianna-Morgante AM and Rosenberg C (2010) Two distinct regions in 2q24.2-q24.3 associated with idiopathic epilepsy. Epilepsia 51:24572460.

Lee JA and Lupski JR (2006) Genomic rearrangements and gene copy-number alterations as a cause of nervous system disorders. Neuron 52:103-121.

Maurice T, Hippert C, Serratrice N, Dubois G, Jacquet C, Antignac C, Kremer EJ and Kalatzis V (2009) Cystine accumulation in the CNS results in severe age-related memory deficits. Neurobiol Aging 30:987-1000.

Scarvie KM, Ballantyne AO and Trauner DA (1996) Visuomotor performance in children with infantile nephropathic cystinosis. Percept Mot Skills 82:67-75.

Tolnay M and Clavaguera F (2004) Argyrophilic grain disease: A late-onset dementia with distinctive features among tauopathies. Neuropathology 24:269-83.

Trauner DA, Chase C, Scheller J, Katz B and Schneider JA (1988) Neurologic and cognitive deficits in children with cystinosis. J Pediatr 112:912-914.

Wamelink MM, Struys EA, Jansen EE, Levtchenko EN, Zijlstra FS, Engelke U, Blom HJ, Jakobs C and Wevers RA (2008) Sedoheptulokinase deficiency due to a $57-\mathrm{kb}$ deletion in cystinosis patients causes urinary accumulation of sedoheptulose: Elucidation of the CARKL gene. Hum Mutat 29:532-536.

\section{Associate Editor: Carlos F.M. Menck}

License information: This is an open-access article distributed under the terms of the Creative Commons Attribution License, which permits unrestricted use, distribution, and reproduction in any medium, provided the original work is properly cited. 